ARTICLE

\title{
Monte Carlo Shielding Calculations for a Spent Fuel Transport Cask with Automated Monte Carlo Variance Reduction
}

\author{
Mitsufumi ASAMI ${ }^{1, *}$, Hidenori SAWAMURA ${ }^{2}$ and Kazuya NISHIMURA ${ }^{2}$ \\ ${ }^{1}$ National Maritime Research Institute, 6-38-1, Shinkawa, Mitaka, Tokyo, 181-0004, Japan \\ ${ }^{2}$ MHI Nuclear Engineering Co., Ltd, 3-1, Minatomirai 3-chome, Nishi-ku, Kanagawa, 220-8401, Japan
}

\begin{abstract}
For the purpose of performing reasonable shielding calculation of a spent fuel transport cask, the use of Monte Carlo methods has been proposed for solving the radiation transport problem on a detailed structure of the transport cask considering fixed neutron sources. A SMIRE (Simplified MCNP-ANISN_W Variance Reduction) system has been developed in the present study, which is possible to generate automatically the lower weight boundary of the weight window for each mesh based on the Consistent Adjoint Driven Importance Sampling (CADIS). Compared with the case of the importance based on the empirical formula, the figure of merit is increased by a factor of 25 . In this system, it is possible to calculate the weight suitable for the distantly-positioned detector point from the fuel effective region to introduce the relaxation factor which relaxes the increase of the particle numbers at the boundary of weight window meshes that are generally caused by the large attenuation of adjoint flux. This system is used for a variety of the radiation transport problems as well as the transport cask.
\end{abstract}

KEYWORDS: CADIS methodology, relaxation factor, weight window, figure of merit, Monte Carlo shielding analysis, empirical formula, adjoint flux

\section{Introduction}

The neutron flux distribution of some complicated shielding structures can be determined precisely by solving the integral Boltzmann transport equation in the Monte Carlo method. Additionally, the Monte Carlo method with continuous-energy cross-section data was considered to be the most accurate method for performing these shielding analyses. Moreover, the use of the Monte Carlo method is exempt from constructing space-energy-angle grids for modeling problems, and hence, no discretization errors are introduced into solving problems. Therefore, the Monte Carlo simulation is relatively straightforward in both physics and geometry modeling.

However, it is difficult to solve radiation transport calculations in practical problems with thick shielding and large source volume by the Monte Carlo method. Thick shielding results in significant attenuation of radiation intensity from the source to the tally. Large source volume causes an additional self-shielding effect for the inner source region. These situations make it more difficult to do sampling effectively of the source variables. These arguments point to a need for the non-analog Monte Carlo techniques are indispensably. Actually, they have been used with great care to prevent getting unreliable results.

To date, a large number of the non-analog Monte Carlo techniques has been developed and improved. In the non-analog Monte Carlo techniques, particles have the statistical weights. The statistical weight of the particle is reduced through physical events such as collision and ab-

*Corresponding author, E-mail:asami@nmri.go.jp sorption. The tracking on the particle is continued until the particle is eliminated from the considered region or until the tracking on the particle reached the number of the particles that have already been set in the calculation. In order to execute simulation with non-analog Monte Carlo techniques efficiently, importance sampling ${ }^{1)}$ is indispensable. In importance sampling, importance functions are selected for suitable variance reduction. After that, many random walks are rationally executed in the objective phase space (space and energy). In particular, a weight window importance parameter $^{2-4)}$ consists of upper and lower boundary for a particle's statistical weight in each phase space region. In the weight window method, the lower weight boundary of each cell which composes the calculation model is set so that the collision density of the particles entering each cell is kept constant. In order to set the variance reduction parameter appropriately, particles entering each cell are to be split or Russian-rouletted correctly.

In the past, the lower weight boundary of the weight window has been determined from the experiences of the Monte Carlo practitioners. This way may lead to inefficient variance reduction parameters of the calculations. For example, if the statistical weights of the source particles are not within the weight window, the particles are split or Russian-rouletted immediately in an effort to bring their weights into the weight window. This event results in unnecessary degradation in computational efficiency. Therefore, respective techniques must be invariably consistent with one another when we conduct the shielding analysis with some variance reduction techniques.

The purpose of the present study is to perform, compare 


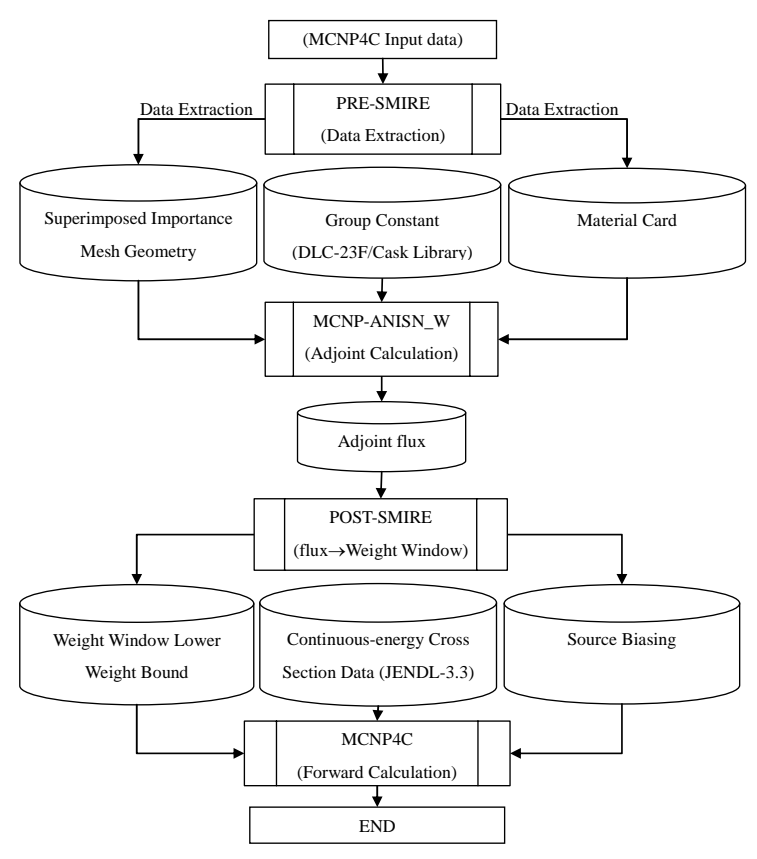

Fig. 1 Calculation flow of the lower weight boundary of the weight window and forward calculation

and analyze the shielding calculations for a transport cask in detail with the variance reduction technique based on the Consistent Adjoint Driven Importance Sampling (CADIS) methodology ${ }^{5-9)}$ and the empirical formula. ${ }^{10)}$ CADIS methodology makes use of the adjoint function that is associated with particle importance which is the contribution of a particle with respect to the objective. The Monte Carlo method with variance reduction shown in the present study should be helpful to those in performing calculations for similar some shielding structures.

\section{SMIRE System}

\section{Description of the SMIRE System}

Figure 1 shows the schematic flow of the SMIRE System to generate the lower weight boundary of the weight window.

MCNP4C code ${ }^{11)}$ is provided with "superimposed importance mesh" which can create the space partition based on mesh that is independent of the geometrical cell. In the present study, the mesh-based weight window parameter generation system has been developed. In this system, complicated cell partitions for the variance reduction are not necessarily required. The lower weight boundary of the weight window in each mesh is determined as follows:

a) Each coordinate of the superimposed importance meshes

is calculated from the mesh information in the MCNP input data.

b) Each of these adjoint fluxes in the superimposed importance meshes is calculated by one-dimensional deterministic code, MCNP-ANISN_W. ${ }^{12)}$ This code makes it possible to solve the one-dimensional neutron transport problem specified by MCNP input data. Figure 2 shows the flow of the MCNP-ANISN_W:

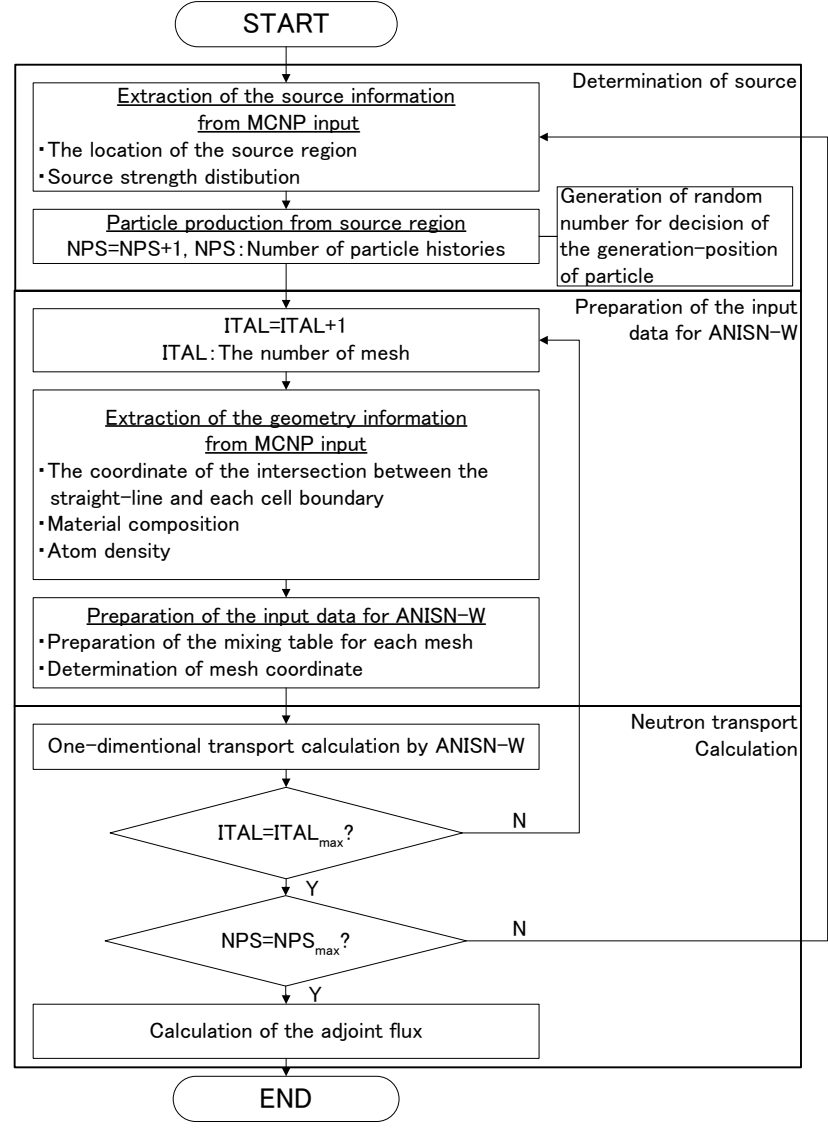

Fig. 2 Flowchart of the MCNP-ANISN_W calculation

(1) A detector locating point is arranged at the center of each mesh which constitutes the geometric form described in MCNP input.

(2) The location of the particle production is stochastically decided from the source region and the source strength distribution described in MCNP input.

(3) Particle energy is stochastically decided from the distribution of source energy spectrum described in MCNP input.

(4) The distance in a straight line between the location of the particle production and the detector location is decided from the geometry data described in MCNP input.

(5) The coordinate of the intersection between the straight line and each cell boundary is decided from the geometry data described in MCNP input. The material composition and the atom density each cell is also obtained from the MCNP input.

(6) The one-dimensional model for the deterministic Sn transport code is made from the calculation conditions acquired from the process (2) to (5). The transport calculation is performed by ANISN-W ${ }^{13)}$ code.

(7) Calculation conditions for ANISN-W are as follows:

Basic geometry form: sphere

Source: Shell source problem 
Left boundary condition: reflection

Right boundary condition: vacuum (no reflection) Outer iteration: 1

(8) The adjoint flux in a certain mesh is obtained by one-dimensional transport calculation.

(9) The adjoint flux of each mesh is obtained by the process from (2) to (8).

(10) The process of the adjoint flux calculation in a certain mesh from (2) to (9) is iterated by the number of particle histories.

(11) The end result of the adjoint flux in a certain mesh is obtained averaged over the number of particle histories.

(12) The adjoint fluxes in all of the mesh are obtained.

c) The code-generated adjoint flux obtained from b) is normalized for each phase space by the adequate method.

d) The reciprocal of the adjoint flux is the lower weight boundary of the weight window of each superimposed importance mesh.

\section{Calculation on Weight Window by the SMIRE System}

The weight window is a variance reduction scheme in which each region of phase space $i$ is assigned an upper and lower weight boundary. ${ }^{14)}$ Particles entering a phase space region with a weight outside the boundaries are either split or Russian-rouletted which is performed to bring their weights into conformity. The weight window can be used in any dimension of phase space. The lower weight boundary of the weight window $W_{L}$ for spatial mesh $i$ and energy group $g$ are calculated from the adjoint flux as follows: ${ }^{15}$

$$
W_{L}(i, g)=\frac{C}{\iint_{V_{i, E_{g}}}\left[\phi^{\dagger}(r, E)\right]^{n_{\mathrm{r}}} d r d E},
$$

where $\phi^{\dagger}(r, E)$ is the adjoint flux at point $r$ and energy $E$, $V_{i}$ and $E_{g}$ represent the volume of spatial mesh $i$ and energy group $g$, and $C$ is the constant for normalization, making $W_{L}$ in the source regions to be a half of the biased weight of source particle. With finite weight window mesh sizes, significant increase of the particle numbers at the boundary of the weight window meshes is generally caused by the large attenuation of adjoint flux. In this case, lowered efficiency of calculations occurs with an increase of CPU time per history. Therefore, the relaxation factor, ${ }^{15)} n_{\mathrm{r}}$ is introduced to improve the calculation efficiency for these cases.

The lower weight boundary of the weight window must be set close to 1.0 near the cell or mesh of the source region because the particle's statistical weight just after being emitted from the source is equal to 1.0. The constant $C$ in Eq. (1) is the adjusting parameter of the reciprocal of the importance. In the present study, the constant $C$ is decided as follows. The adjoint flux at energy group $g$ averaged over the real source region is given by Eq. (2).

$$
\overline{\phi^{s}}\left(E_{g}\right)=\sum_{i} \frac{\phi_{i}^{s}\left(E_{g}\right)}{V_{i}}
$$

Where, $V_{i}$ and $E_{g}$ represent the volume of spatial mesh $i$
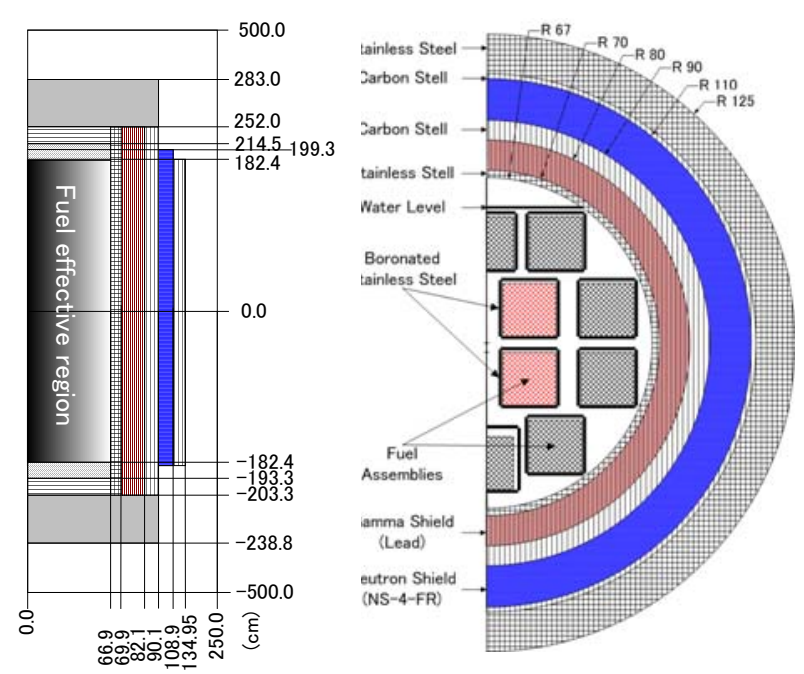

Fig. 3 Calculation geometry of the transport cask and adjoint source region ${ }^{17-18)}$

and energy group $g$, respectively. $\phi_{i}^{s}\left(E_{g}\right)$ is the adjoint flux at energy group $g$ and spatial mesh $i$.

Here, the lower weight boundary of the weight window is set by the use of $\overline{\phi^{s}}\left(E_{g}\right)$ as follows: Let the energy group corresponding to the maximum of the source energy spectrum distribution before biasing be $E_{\mathrm{max}}^{\mathrm{def}}$. The lower weight boundary of the weight window corresponding to the energy $E_{\max }^{\text {def }}$ was assumed to be the standard of the lower weight boundary of the weight windows of other energy groups; the lower weight boundary of the weight window on the source region was set as the reciprocal of the adjoint flux at the energy $E_{\max }^{\text {def }}$ averaged over the real source region, $1 / \overline{\phi^{s}}\left(E_{\max }^{\mathrm{def}}\right)=0.5$. In the case of the transport cask such as containing the spent fuels, the energy group $E_{\max }^{\text {def }}$ is $1.11-1.83 \mathrm{MeV}$ with the energy group structure of DLC-23/CASK. ${ }^{16)}$

\section{Validation of SMIRE System}

The SMIRE system is tested by applying to an analytical model of a cask, as shown in Fig. 3. The model was prepared in considerable detail, especially in the fuel basket. The main specifications of the cask are as follows; total weight is 115.0 tons, outer diameter is $2.6 \mathrm{~m}$ and height is $6.3 \mathrm{~m}$, main structure is carbon steel, the fuel basket is composed of stainless steel both with and without boron, lead is used for a gamma-ray shield and NS-4-FR resin is used for a neutron shield, and it has cooling fins made of stainless steel. It is possible to install 14 bundles of pressurized water reactor spent-fuel assemblies. ${ }^{17-18)}$ Figure 3 also shows the source region for the calculation of the adjoint flux. Since the source intensity of neutrons in a spent fuel assembly depends strongly on the specific burn-up distribution in the axial direction, the burn-up distribution was taken into account in the present calculation. A peaking factor of 1.15 was assumed for the middle part of 10/12 of all fuel assemblies. The effective multiplication factor, $k_{\text {eff }}$, of the cask containing 14 PWR assemblies was calculated by the KENO 


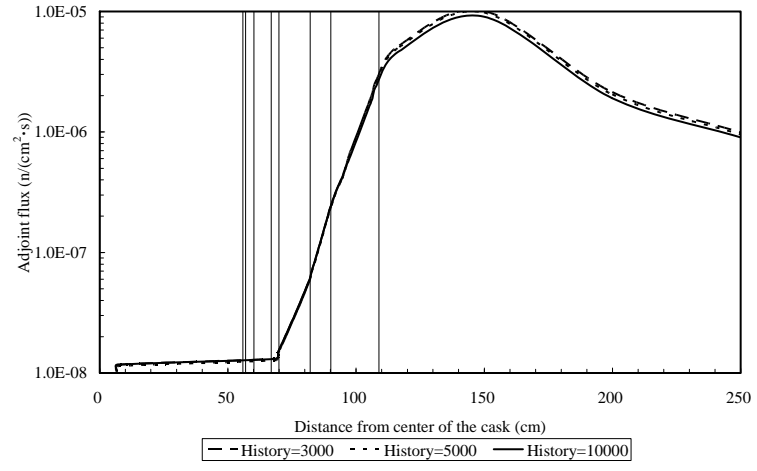

(Adjoint flux at $14.9 \mathrm{MeV}$ )

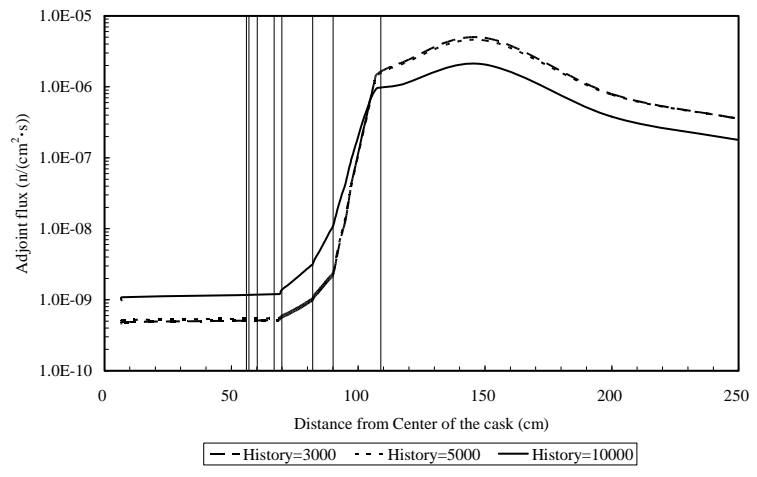

(adjoint flux at energy $1.11 \mathrm{MeV}$ )

Fig. 4 Comparison of adjoint flux at energy $14.9 \mathrm{MeV}$ and $1.11 \mathrm{MeV}$

V.a code, ${ }^{19)}$ and a $k_{\text {eff }}$ of 0.63 was used to obtain the neutron source intensity of the cask.

DLC-23/CASK library was used for all adjoint flux calculations. The weight window parameters, source energy biasing and the dose-rate conversion factor were given in the energy group structure of DLC-23/CASK library. The dose-rate conversion factor ${ }^{20)}$ was used for the energy spectrum for the calculation of the adjoint flux. In addition, the final Monte-Carlo shielding calculation was performed with the Japanese Evaluated Nuclear Data Library, JENDL-3.3. ${ }^{21)}$

Figure 4 shows the calculation result of the adjoint flux for each mesh of the calculation model with a different number of particle histories $(3,000,5,000$ and 10,000 , respectively). The fuel effective region was equally divided into three in accordance with axial direction. The detector location was set at $(x, y, z)=(0,135,0)$. As shown Fig. 4, number of particle histories has an insignificant effect on the calculation result of the adjoint flux above $14 \mathrm{MeV}$. On the other hand, it is shown that the distribution of the adjoint flux below $1.11 \mathrm{MeV}$ depends strongly on number of particle histories. Analysis shows that number of particle histories requires at least 10,000 to calculate the adjoint flux of each mesh in the shielding configuration such as the transport cask. Therefore, the adjoint flux was conservatively calculated with a history number of 10,000 for the transport cask.

\section{Results and Discussion}

In the present study, the relaxation factor $n_{\mathrm{r}}$ was intro-

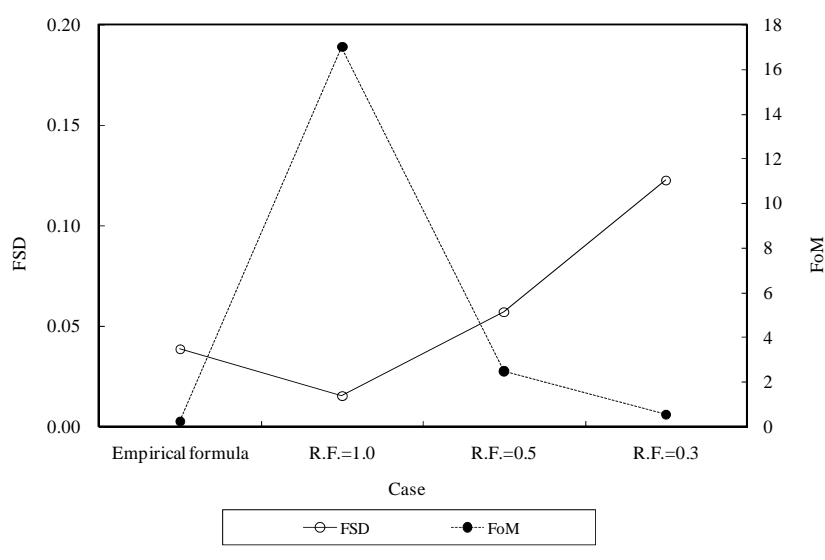

Fig. 5 FoM for the neutron dose rate at the side surface of the cask calculated with the lower weight boundary of the weight window that is generated by using a different relaxation factor. R.F. denotes the relaxation factor.

duced ${ }^{15)}$ which relaxes the attenuation of the adjoint flux among adjacent meshes:

$$
W_{i}^{\text {low }} \propto \frac{1}{\phi^{\dagger}} \rightarrow\left(W_{i}^{\text {low }}\right)^{n_{\mathrm{r}}} \propto\left[\frac{1}{\phi^{\dagger}}\right]^{n_{\mathrm{r}}}, n_{\mathrm{r}} \leq 1.0
$$

Where, $W_{i}^{\text {low }}$ is the lower weight boundary and $\phi^{\dagger}$ is the adjoint flux.

The calculation of the lower weight boundary was performed in cases of $n_{\mathrm{r}}=1.0,0.5,0.3$, respectively, and then the shielding calculation was performed by using these lower weight boundaries of the weight window. The detector location was set at $(x, y, z)=(0,135,0)$.

Figure 5 shows the result of the computational efficiency (Figure of Merit: FoM) and the fractional standard deviation. For a detector point near the axial center of the fuel effective length in the transport cask, the computational efficiency with the relaxation factor $n_{\mathrm{r}}=1.0$ is better than with $n_{\mathrm{r}}<1.0$. It is shown that the calculation is correctly performed for transport processes based on the deterministic adjoint flux obtained from these results.

The computational efficiency based on the lower weight boundary of the weight window that was generated by the SMIRE system was compared to that based on the importance that is based on the empirical formula. The calculation result is shown in Fig. 6. The horizontal axis is the spatial coordinates $(x, y, z)$ and the longitudinal axis is the FoM or the fractional standard deviation. This figure shows that the computational efficiency based on the adjoint flux by using the SMIRE system is better than that based on the empirical formula at the center of the fuel effective length. Compared with the case of the importance based on the empirical formula, the figure of merit is increased by a factor of 25 .

The computational efficiency is very low at the point away from the fuel effective length. Here, the calculation was performed by the lower weight boundary of the weight window based on the adjoint flux obtained from the adjoint source arranged around the top-end of the transport cask. The detector location was set at $(x, y, z)=(0,135,0)$. Figure 7 shows the calculation result. As shown in Fig. 7, in the 


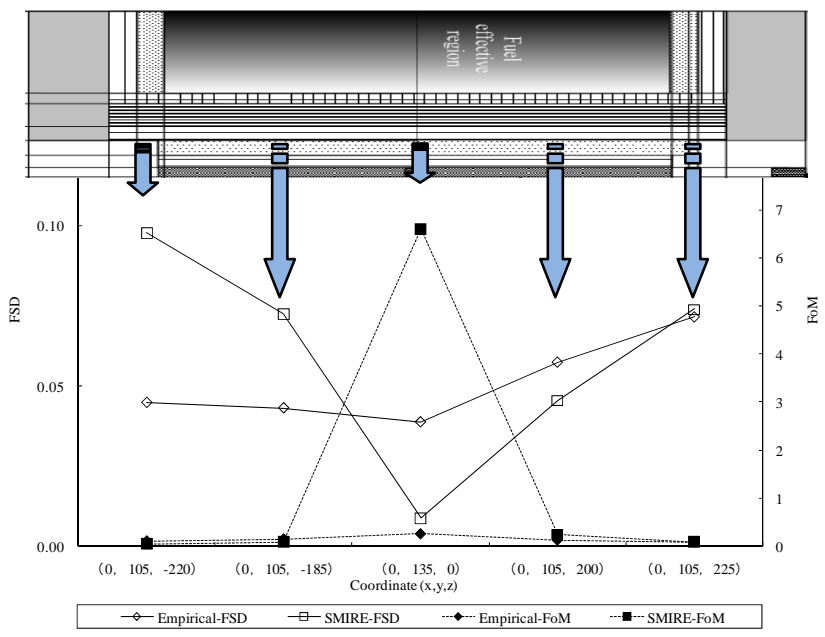

Fig. 6 The fractional standard deviation (FSD) and the figure of merit (FoM) in an calculation point on the surface of the transport cask. The coordinates on the horizontal axis represent the coordinates of the calculation point. Each calculation point corresponds with the position of the transported cask as illustrated in this figure.

case of optimum relaxation factor is 0.5 , the figure of merit is increased by a factor of 50 compared to the case based on the empirical formula. This is because the SMIRE system makes the one-dimensional model, the straight line distant from the neutron generation location in the source region to the point detector. In the SMIRE system, it is possible to calculate a distantly- positioned detector point from the fuel effective region to introduce the relaxation factor. If the geometric condition can be modeled by one-dimensional form such as the side surface of the cask, the adjoint flux which is useful for the variance reduction is obtained without respect to the relaxation factor $\left(n_{\mathrm{r}}=1.0\right)$. If the detector is located in the side part of the transport cask, and at some distance from the both ends of the fuel effective length shown in Fig. 6, the use of the one-dimensional model has limited flexibility. In the case of the spent fuel transport cask discussed in this paper, the limitation of the one-dimensional model is relaxed effectively by the relaxation factor such as $n_{\mathrm{r}}=0.5$. The adjoint flux is corrected appropriately by the relaxation factor, which is useful for the variance reduction. The optimum value of the relaxation factor for the spent fuel transport cask is obtained on a case-by-case basis. Each spent fuel transport cask has similarities with the geometric condition and source information. Therefore, the relaxation factor $n_{\mathrm{r}}=0.5$ has applicability to various casks for the spent fuel.

\section{Conclusion}

This study demonstrated the variance reduction with the adjoint flux calculated with one-dimensional discrete ordinate method for the Monte Carlo shielding calculations of the spent fuel transport cask. The SMIRE system has been developed for automatically calculating the parameters of weight window and source biasing for precise shielding calculations. Compared with the case of the importance based

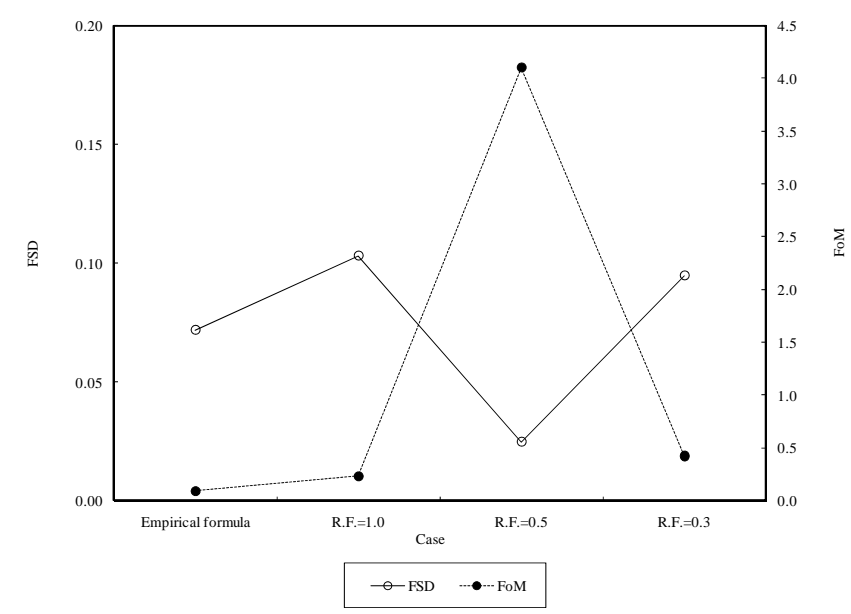

Fig. 7 Standard deviation and Figure of merit at a point away from the fuel effective length. R.F. denotes the relaxation factor.

on the empirical formula, the figure of merit is increased by a factor of 25. If the detector is located in the side part of the transport cask, and at some distance from the both ends of the fuel effective length, the limitation of the one-dimensional model is relaxed effectively by the relaxation factor such as $n_{\mathrm{r}}=0.5$. The adjoint flux is corrected appropriately by the relaxation factor, which is useful for the variance reduction. As a result, the figure of merit is increased by a factor of 50 compared to the case based on the empirical formula. The SMIRE system is useful for the calculation of the dose rate for any point around the cask regardless of experience of the practitioner.

\section{Acknowledgment}

This study was financially supported by the Budget for Nuclear Research of the Ministry of Education, Culture, Sports, Science and Technology, based on the screening and counseling by the Atomic Energy Commission.

\section{References}

1) M. H. Kalos, "Importance Sampling in Monte Carlo Shielding Calculations,” Nucl. Sci. Eng., 16, 227 (1963).

2) T. E. Booth et al., "Importance Estimation for Monte Carlo Calculations,” Nucl. Technol./Fusion, 5, 91 (1984).

3) T. E. Booth, "Automatic Importance Estimation in Forward Monte Carlo Calculations,” Trans. Am. Nucl. Soc., 41, 308 (1982).

4) J. S. Hendricks, "A Code-Generated Monte Carlo Importance Function,” Trans. Am. Nucl. Soc., 41, 307 (1982).

5) J. C. Wagner, A. Haghighat, “Automated Variance Reduction of Monte Carlo shielding Calculations Using the Discrete Ordinates Adjoint Function,” Nucl. Sci. Eng., 128, 186-208 (1998).

6) K. Ueki et al., "Continuous Energy Monte Carlo Analysis of Neutron Shielding Benchmark Experiments with Cross Sections in JENDL-3,” J. Nucl. Sci. Technol., 30, 339 (1993).

7) J. F. Briesmeister (Ed.), MCNP-A General Monte Carlo $\mathrm{N}$-Particle Transport Code Version 4C, LA-13709-M, Los Alamos National Laboratory (LANL) (2000).

8) M. Asami, S. Ohnishi, K. Kawakami, T. Matsumoto, N. Odano, "Rational Shielding Ability Evaluation for a Modular Type Interim Storage Facility,” Trans. At. Energy Soc. Japan, 6[4], 
460-475 (2007), [in Japanese].

9) R. K. Disney, J. Jedruch, R. G. Soltesz, R. S. Kaiser, S. L. Zeigler, ANISN-W, WANL-TME-2778, Westinghouse Astronuclear Laboratory (1971).

10) J. S. Hendricks, “A Code-Generated Monte Carlo Importance Function,” Trans. Am. Nucl. Soc., 41, 307 (1982).

11) S. Mitake, O. Sato, H. Tsunoda, "Application of Biasing Optimization Techniques to Monte Carlo Shielding Analysis of a Transport Cask,” Proc. MC2000 Lisbo, October 23-26, 2000, Springer-Verlag, 859 (2001).

12) CASK-81: 22Neutron, 18Gamma-Ray Group, P3 Cross Sections for Shipping Cask Analysis, DLC-23, Radiation Safety Information Computational Center, Oak Ridge National Laboratory, (1974).

13) K. Ueki, K. Sasaki, "Radiation Shielding Analysis of a Spent Fuel Transport Cask with an Actual Configuration Model Using the Monte Carlo Method - Comparison with the Discrete Ordinates Sn Method," Proc. The 14th Int. Conf. on the Packaging and Transportation of Radioactive Materials (PATRAM'04) (2004).

14) Y. Nakahara, K. Suyama, T Suzaki, Technical Development on Burn-up Credit for Spent LWR Fuels, JAERI-Tech 2000-071 Japan Atomic Energy Research Institute (JAERI) (2000), [in Japanese].
15) L. M. Petrie, N. F. Landers, KENO V.a: An Improved Monte Carlo Criticality Program with Super-grouping, NUREG/CR-02000, Rev.6, Vol.2, Sec. F11, ORNL/NUREG /CSD-2/R6, Oak Ridge National Laboratory (ORNL) (1998).

16) Atomic Energy Society of Japan, Radiation Dose conversion Coefficients for Radiation Shielding Calculations: 2004, AESJ-SC-R002 (2004), [in Japanese].

17) K. Shibata et al., "Japanese Evaluated Nuclear Data Library Version 3 Revision-3: JENDL-3.3,” J. Nucl. Sci. Technol., 39, 1125 (2002).

18) A. Haghighat, J. C Wagner, "Monte Carlo Variance Reduction with Deterministic Importance Functions," Prog. Nucl. Energy, 42[1), 25-53 (2003).

19) A. Haghighat, J. C. Wagner, "Application of $A^{3} M C N P$ to Radiation Shielding Problems," Proc. the Int. Conf. on Monte Carlo for Radiation Physics, Particle Transport Simulation and Applications, Lisbon, Portugal, October 23-26, (2000).

20) M. W. Mickael, "A Fast, Automated, Semideterministic Weight Windows Generator for MCNP,” Nucl. Sci. Eng., 119, 34 (1995).

21) J. S. Tang, T. J. Hoffman, "Monte Carlo Shielding Analyses Using an Automated Biasing Procedure," Nucl. Sci. Eng., 99, 329 (1988). 